\title{
Clinical and Histologic Manifestations of a Novel Rectus Femoris Myotendinous Junction Injury in Rats
}

\author{
K. J. Sikes ${ }^{1 *}$, K. M. Andrie ${ }^{2 *}$, A. McConnell', S. Wist ${ }^{2}$, S. Smith ${ }^{1}$, B. Cole ${ }^{3}$, D. D. Frisbie ${ }^{1}$, \\ K. S. Santangelo ${ }^{2}$ \\ 1 Department of Clinical Sciences, Colorado State University, Fort Collins (CO), U.S.A. \\ 2 Department of Microbiology, Immunology and Pathology, Colorado State University, Fort Collins (CO), U.S.A. \\ 3 Department of Orthopaedic Surgery, Rush University Medical Center, Chicago (IL), U.S.A. \\ * These authors are co-first authors
}

\section{CORRESPONDING AUTHOR:}

Kelly S. Santangelo

Department of Microbiology,

Immunology and Pathology

Colorado State University

1682 Campus Delivery Fort Collins (CO)

80523-1621, U.S.A.

E-mail: Kelly.Santangelo@colostate.edu

DOI:

10.32098/mltj.04.2021.01

LEVEL OF EVIDENCE: 1B

\begin{abstract}
SUMMARY
Background. Animal models of muscle injury have primarily relied on methods which do not mimic the chronic scarring that typically occurs adjacent to the myotendinous junction (MTJ). The goal of this study was three-fold: (i) to create a strain-induced in vivo model of rectus femoris MTJ injury in rats; (ii) to document clinical manifestations of injury using longitudinal tracking of individual animals via voluntary and compulsory (treadmill) mobility analyses and (iii) to validate and assess the model for persistent scarring through serial histologic assessment and development of a semi-quantitative grading scheme to characterize injury response over time.

Methods. Strain-induced MTJ injury was generated in male Sprague Dawley rats via needle tension directed along the transverse axis between the rectus femoris muscle and distal tendon that attaches to the patella. Animals received mobility assessments (gait analysis using a DigiGait Treadmill System and weight bearing using a Tekscan Rodent Walkway System) at days 0, 1, 3, 6, 13, 20, and 27 of the experimental protocol. Rats were euthanized at $1,3,7,14$, and 28 days post-injury ( $\mathrm{n}=6$ rats per time-point) and hindlimbs were processed for histology.

Results. Significant changes in locomotor parameters included injured and contralateral limb paw area, max dA/dt (limb deceleration/breaking time), stride time, stance time, force time impulse, and fore/hind symmetry, and injured limb maximum force. The most significant and consistent histologic finding was a pathologic fibrotic adhesive lesion at the muscle and tendon interface along the proximal aspect of the patella just distal to the injury site. This lesion was composed of reactive fibroblasts, disorganized collagen fibers, vascular profiles, and a myxomatous ground substance stroma. Conclusions. This work is the first to characterize the clinical and pathologic development of a chronic model of rectus femoris MTJ injury, which resulted in altered mobility likely caused by a strain-induced fibrotic scar along the anterior patella. Notably, both the functional and pathologic changes recapitulated the course of injury progression similar to what is described in humans. This work provides a unique model to study MTJ injury mechanisms for the identification of enhanced treatment options for patients who suffer from activity-related muscle conditions.
\end{abstract}

\section{KEY WORDS}

Animal model; gait analysis; histopathology; myotendinous junction; quadriceps rectus femoris. 


\section{ABBREVIATIONS}

MTJ: Myotendinous Junction

EDTA: Ethylenediaminetetraacetic Acid

$\mathrm{H} \& \mathrm{E}$ : Hematoxylin and Eosin

\section{BACKGROUND}

The myotendinous junction (MTJ) is as a highly specialized transition zone composed of many interacting tendinous and muscular filaments that contribute to physiologic adhesion and anchoring (1). This site is responsible for the transmission of force between the muscle, tendon, and bone, which ultimately dictates skeletal movement and gait patterns. As the connection between two different tissue types, the MTJ represents the weakest link along the greater muscle-tendon unit, making it highly vulnerable to tension-induced impairment $(2,3)$. Activities that induce eccentric loading, such as high speed running and kicking, pose an increased risk of MTJ injury (4). While the mechanical contributions of MTJ strains (stretch) during eccentric loading are fairly well described $(4,5)$, little is known about the clinical (gait and weight bearing parameters) or structural (histopathologic) responses to injury.

The quadriceps, hamstrings, and gastrocnemius muscles are uniquely prone to MTJ injury due to their superficial location and extension across two joints $(2,6)$. Indeed, lower limb orthopedic injuries affect 25-48\% (7-9), 62.4\% (10), and $32.3 \%$ (11) of military, athletic, and occupational populations, respectively, with strains of the aforementioned muscles accounting for the majority of these conditions. Depending on the severity of MTJ injury, patients present with swelling, tenderness, and pain (12). Due to the complicated structural organization and cell signaling at the MTJ, endogenous healing for structural and functional recovery is difficult. Current treatment regimens for MTJ injuries remain conservative and include the RICE method (rest, ice (cold), compression, elevation) followed by gradual exposure to physical exercise modalities (2). If not addressed, persistent scarring within the muscle directly adjacent to the MTJ can lead to reduced flexibility, weakness, and muscle atrophy, making previous injuries a leading risk factor associated with recurrence $(7,13$, 14). Therefore, the controlled study of MTJ injury progression in a clinically relevant pre-clinical model may allow for the development of novel therapeutics and treatment strategies for patients who suffer from such injuries.

Animal models of muscle injury include chemical (15), traumatic $(16,17)$, acute high intensity exercise $(18)$, contusion (19-21), contraction (22), and ischemia-reperfusion (23) methods (for a summary of published models see appendix 1). While valuable, the majority of current models do not specifi- cally target the MTJ, a common site of clinical strain-induced activity-related injuries $(2,3)$. Recently, a model of gastrocnemius MTJ injury was developed in the rat (24). Through a needle core puncture directed along the transverse axis of the MTJ, grade I-II muscle lesions were generated as early as 1 day post-injury. Of note, muscle lesions in this model resolved by 26-46 days post-injury. Similarly, models that utilize mechanically and tetanically induced strain injuries $(25,26)$ replicate acute muscle damage near the MTJ without longer term lesion development. While these provide suitable models of inherent muscle regeneration and healing, a model that recapitulates the persistent scarring seen clinically would be advantageous for testing new methods of clinical management and reduction of re-injury rates. Therefore, the development of a chronic animal model is needed to better characterize factors leading to high re-injury rates and investigate interventions that may expedite patient recovery.

While gastrocnemius MTJ injury (24) has been studied to date, no published reports exist that employ an animal model of quadriceps MTJ injury. The most commonly reported site of quadriceps MTJ injury in humans is the distal MTJ of the rectus femoris muscle belly where it inserts on the proximal aspect of the knee joint $(4,27,28)$. More specifically, given its short length, MTJ injuries of the rectus femoris usually occur near the osteotendinous junction approximately $2 \mathrm{~cm}$ from the insertion of the quadriceps tendon on the patella (29). Indeed, as a bipennate muscle with short muscle fibers, a steep oblique tendon attachment, and a large MTJ transition zone (30), the rectus femoris is highly susceptible to strain during stretching (31). Owing to this, the goal of this study was to create a strain-induced in vivo model of rectus femoris MTJ injury in rats. Findings were characterized by: (i) documenting clinical manifestations and progression of injury using longitudinal tracking of individual animals via voluntary and compulsory (treadmill) mobility analysis; and (ii) validating and assessing the model for persistent scaring through temporal histologic assessment and establishment of a semi-quantitative grading scheme to characterize injury response over time. The controlled study and application of this model of MTJ injury may allow for the development of novel therapeutics and treatment strategies for patients who suffer from such injuries.

\section{MATERIALS AND METHODS}

\section{Animals}

All procedures were approved by the University's Institutional Animal Care and Use Committee (Protocol \#16-6927A),were performed in accordance with the NIH Guide for the Care and Use of Laboratory Animals, and were in accordance with the MLTJ guidelines (32). Thir- 
ty (30) male Sprague Dawley rats were purchased from a commercial vendor (Charles River Laboratory, Wilmington, MA) at 70 days of age and allowed to acclimate to the vivarium for 14 days. A single sex was chosen to standardize the model as (i) injury severity was dictated by the needle size relative to the targeted region, (ii) rectus femoris injury in males is better characterized clinically, and (iii) due to their size, males offered a larger targeted area for initial model development. Animals were monitored daily by a veterinarian. All rats were housed singly in solid bottom cages with corncob bedding and were maintained at $22-24{ }^{\circ} \mathrm{C}$ on a 12 $\mathrm{h}$ light/dark cycle. Commercially available irradiated water and food were available ad libitum during the experiments.

\section{Rectus Femoris Injury at the Myotendinous Junction}

Animals were anesthetized using a mixture of isoflurane and oxygen $(2-4 \%)$ and both limbs were shaved and surgically prepped using chlorohexidine and isopropyl alcohol. The surgical region of interest was visualized via a $1 \mathrm{~cm}$ incision along anterior aspect of the knee. Strain-induced MTJ injury was generated on the right hind limb via needle tension (using an 18G hypodermic needle) that was directed along the transverse axis between the rectus femoris muscle and quadriceps tendon (figure $1 \mathrm{~A}$ and see appendix 2). Specifically, this injury focused on tissue plane separation and stretching (6) to induce strain between the tendon/muscle and surrounding structures, as contrasted to a coring needle puncture biopsy utilized in previous reports (24). For matched comparisons and clinical translatability, the left limb was utilized as a sham surgery control, receiving an anterior superficial surgical incision without subsequent needle injury. Rats were randomized to end-points and sacrificed at either day $1,3,7,14$, and 28 days post-injury ( $\mathrm{n}=6 /$ timepoint; see figure $\mathbf{1}$ B) in accordance with approved protocols $\left(\mathrm{CO}_{2}\right.$ inhalation with confirmatory cervical dislocation).

\section{Mobility assessments}

The 28 day time-point rats underwent compulsory gait analysis using a DigiGait Treadmill System (Mouse Specif-
A.
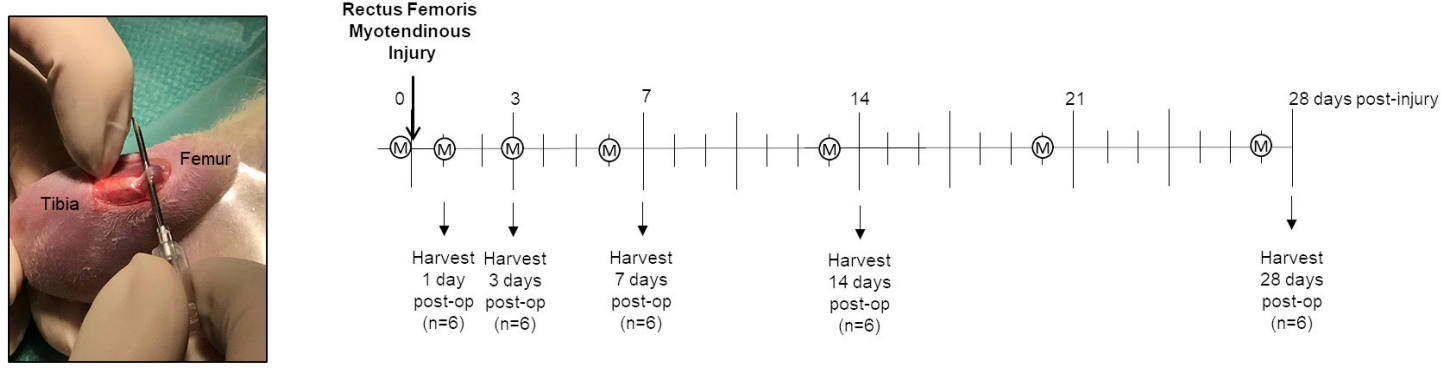

c.
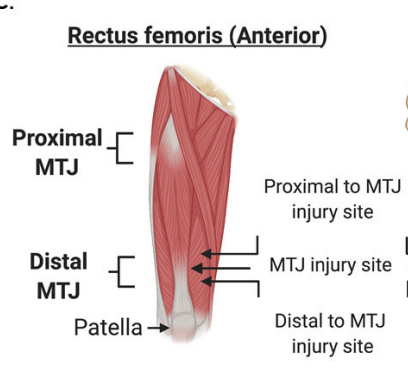
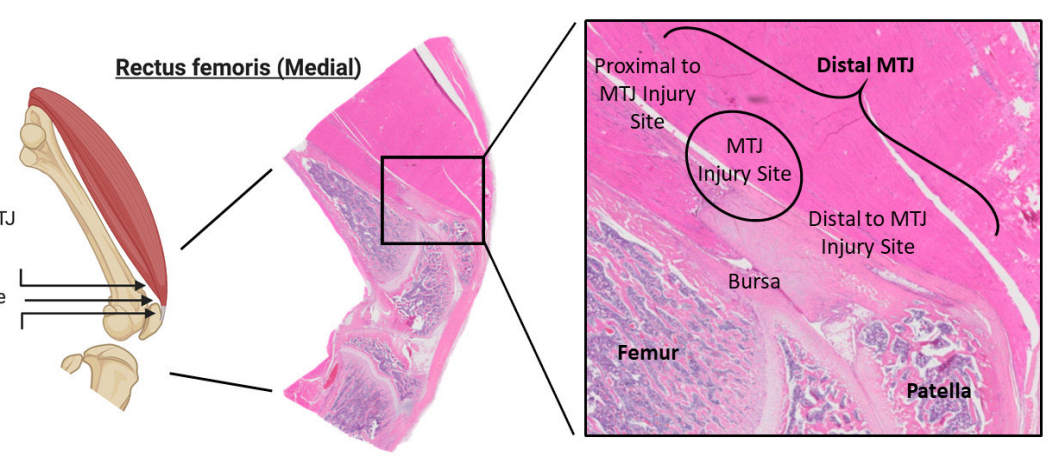

Figure 1. (A) Strain induced MTJ injury generated via $18 \mathrm{G}$ needle tension directed along the transverse axis of the MTJ. (B) Experimental time-course following injury. Rats were euthanized at 1, 3, 7, 14, and 28 days post-injury ( $\mathrm{n}=6 /$ time-point) and their limbs taken for histology. Animals harvested for the 28 day time-point received mobility assessments (marked with $\mathrm{M}$ ) at days $0,1,3,6,13,20$, and 27 of the experimental protocol. (C) Anatomic location of quadriceps distal MTJ injury (from BioRender) with a representative H\&E stained section demonstrating a naïve/control MTJ. The injury site in this model occurred where the MTJ anchors the distal quadriceps muscle belly to the proximal patellar tendon. 
ics, Framingham, MA) and voluntary weight bearing using a Tekscan Rodent Walkway System (South Boston, MA) on Days $0,1,3,6,13,20$, and 27 of the experimental protocol. Prior to start of the experiments, rats were acclimated to both systems over one week. For gait analysis, rats were run for three consecutive replicates per time-point on a flat treadmill at $30 \mathrm{~cm} / \mathrm{sec}$, and videos were analyzed for 22 gait parameters including stride length, \% swing stride, $\%$ stance stride, $\%$ brake stride, $\%$ propel stride, and stride frequency. For weight bearing, rats were allowed to walk voluntarily over the Tekscan walkway for 3 times/ day, and videos were analyzed for 12 weight bearing and gait parameters including maximum force, force time impulse, and maximum force symmetry values (front/hind, left/right, left front/right front, left hind/right hind). For both gait analysis and weight bearing, the three runs taken at each time-point were averaged and utilized for statistical comparisons. For information regarding how specific mobility parameters were calculated see equipment specific manuals.

\section{Histology and Histopathology}

Following euthanasia, hind limbs were removed at the coxofemoral joint and placed into 10\% neutral buffered formalin for 48 hours. Limbs were then transferred to a $10 \%$ solution of ethylenediaminetetraacetic acid (EDTA) at $\mathrm{pH} 7$ for decalcification. EDTA was replaced twice weekly for 8-10 weeks. Limbs were trimmed in the sagittal plane, routinely processed and embedded, sectioned at $5 \mathrm{um}$, and stained with hematoxylin and eosin (H\&E). Histologic sections were evaluated by one author blinded to the groups. A semi-quantitative grading scheme was developed to characterize injury-related histologic changes associated with needle puncture. The three sites assessed included: 1) the MTJ at the needle insertion/injury site; 2) the MTJ immediately distal to the injury site; and 3) the MTJ immediately proximal to the injury site (figure $\mathbf{1} \mathbf{C}$ ). At each location, the following parameters were scored: 1) lesion size, 2) degree of fibrosis (a measure of scarring), 3) ground substance (myxomatous degeneration), and 4) vascularity, with each having an allowable score of 0-3. Additionally, secondary changes were documented including: bursal cellularity/vascularity, and the presence or absence of reactive synovium and joint space effusion, with each having an allowable score of $0-2$. Animals were assessed for the aforementioned histologic changes at days $1,3,7,14$, and 28 post-surgery ( $n=6$ males per group). Based on the degree of injury-associated pathology in each individual animal, the minimum possible score was 0 and the maximum possible score was 41 . The histopathology grading scheme is provided in appendix 3 .

\section{Statistics}

The experimental sample size (6 animals per time-point) was calculated using GPower Version 3.1.1 (33). Specifically, an a-priori power analysis was conducted using pilot gait data (stride length) obtained using this injury model in rats. This power analysis resulted in a power of $80 \%$, using a $95 \%$ confidence interval and typical standard deviation of 1.00 within groups. All post-hoc statistics were conducted in GraphPad Prism 8.3.0 (San Diego, CA). For gait analysis and weight bearing parameters that quantify individual limb changes (stride length, \% swing stride, etc.), groups were compared using a repeated measures, mixed effects analysis with limb (injured right hind, sham left hind, right fore, and left fore), time (days $0,1,3,6,13,20$, and 27 post-op) and limb/time interaction factors. Tukey's posthoc tests were used to compare individual groups when the factors (limb, time, and limb/time) showed significance. For gait analysis and weight bearing parameters that demonstrate the relationship between limbs (e.g. stance factor, step angle, overlap distance, paw placement positioning, and weight bearing symmetry parameters), a repeated measures One-Way ANOVA with Tukey's post-hoc tests was utilized. For all graded pathology parameters, timepoints were compared using a Kruskal-Wallis non-parametric One-Way ANOVA with Dunn's post-hoc tests. For correlation between parameters, a Pearson Correlation Analysis was conducted. Significance was set to $\mathrm{p}<0.05$ for all comparisons.

\section{RESULTS}

\section{Mobility assessments}

For treadmill-based gait analysis parameters, paw area significantly increased over time for both the injured right hind and sham left hind limbs (figure $2 \mathrm{~A}$ ). This was associated with a greater Max $\mathrm{dA} / \mathrm{dt}$ (increased deceleration or breaking time during the stance phase) over time for both hind limbs (figure 2 B). Representative paw area output curves qualitatively demonstrate altered stance phase parameters up to 27 days post-injury (figure 2 C). No statistically significant differences were found for stride length $(\mathrm{cm})$, \%swing stride, \%brake stride, \%brake stance, \%propel stride, \% propel stance, \%stance stride, stance/swing, stride frequency (steps/sec), absolute paw angle (degrees), min $\mathrm{dA} / \mathrm{dt}\left(\mathrm{cm}^{2} / \mathrm{sec}\right)$, gait symmetry, ataxia coefficient, midline distance $(\mathrm{cm})$, axis distance $(\mathrm{cm})$, stance width $(\mathrm{cm})$, stance factor, step angle (degrees), overlap distance $(\mathrm{cm})$, or paw placement positioning $(\mathrm{cm})$. 

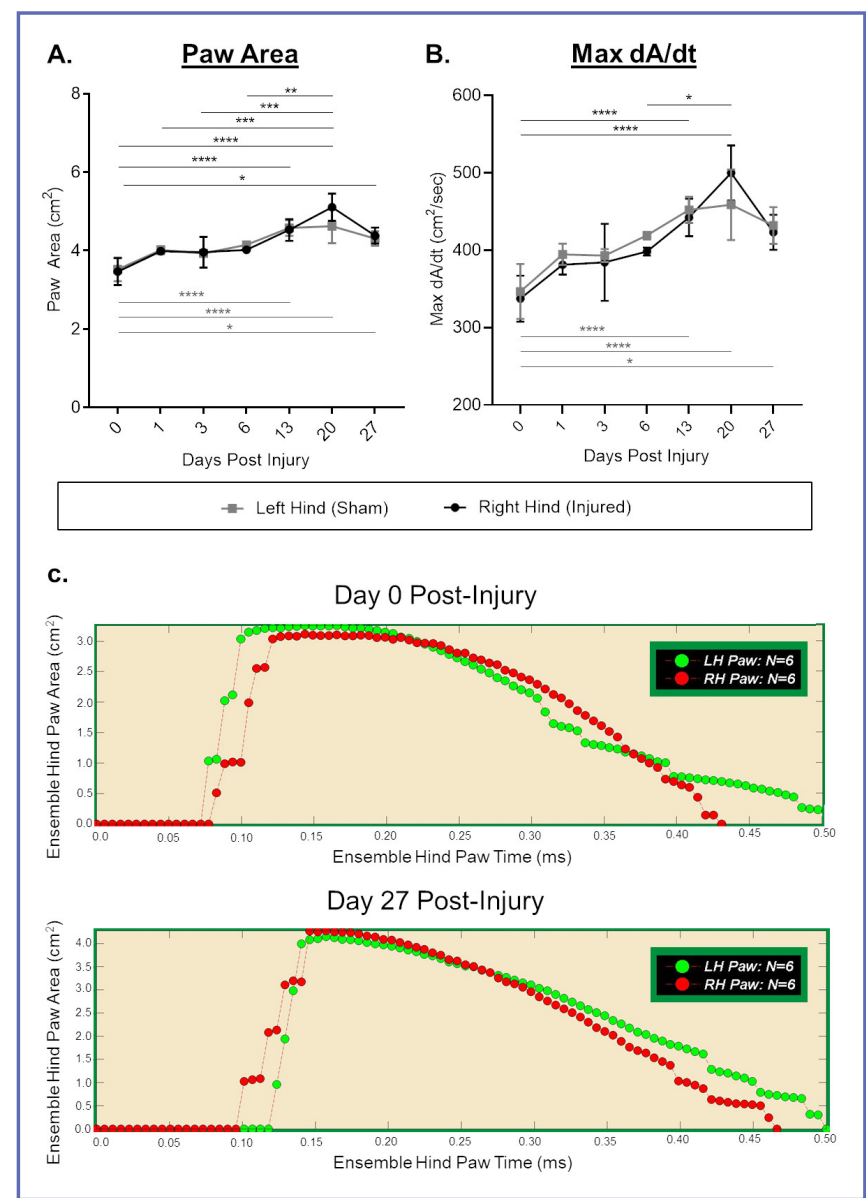

Figure 2. Treadmill based gait analysis parameters (Digigait Treadmill System) following quadriceps MTJ injury in the right hind limbs of rats. Significant spatiotemporal parameters include (A) paw area $\left(\mathrm{cm}^{2}\right)$ and (B) Max dA/dt $\left(\mathrm{cm}^{2} / \mathrm{sec}\right)$. Significance differences over time marked $\left({ }^{\star} p<0.05,{ }^{* *} p<\right.$ $0.01,{ }^{* \star *} p<0.001,{ }^{* \star \star *} p<0.0001$ ) with right hind (injured) differences in black at the top and left hind (sham) differences in grey at the bottom. No statistically relevant differences between hind limbs were noted. Values are represented as Mean +/- Standard Deviation. (C) Representative output paw area curves demonstrating altered stance phase parameters at 27 days post-injury.

For voluntary weight bearing parameters, a decrease in maximum force was seen in the injured right hind limb out to 27 days post-injury, but not the sham left hind limb (figure $3 \mathrm{~A}$ ). Importantly, no appreciable correlation $\left(\mathrm{p}=0.36, \mathrm{R}^{2}=0.02\right)$ was found between maximum force and maximum velocity, demonstrating that animal speed did not dictate force measurements. Increases in the force time impulse increased for both the injured right hind and sham left hind limbs; however, this normalized by 27 days post-injury (figure 3
B). Interestingly, altered hind limb loading was associated with an increase to the fore/hind limb symmetry value, suggesting decreased hind limb loading was associated with increased fore limb loading (figure $3 \mathrm{C}$ ). In contrast to obligatory treadmill walking at a set speed (where no differences in temporal parameters of the stride and stance phases were appreciated), voluntary walking demonstrated a significantly increased stride time (figure 3 D) and stance time (figure 3 E) out to 27 and 20 days post-injury, respectively, with peaks at 6 days post-injury. These changes were associated with a decreased stride velocity (figure $3 \mathbf{F}$ ) at 6 days post-injury with normalization by 27 days post-injury. No significant differences were found for maximum peak pressure $(\mathrm{MPa})$, maximum force left/right symmetry, maximum force left hind/right hind symmetry, stride length $(\mathrm{cm})$ swing time (sec), stride acceleration $\left(\mathrm{cm}^{2} / \mathrm{sec}\right)$.

\section{Histology and Histopathology}

For rectus femoris MTJ injury, a novel grading scheme was developed that separately and cumulatively assessed the following regions of the MTJ: 1) needle injury site; 2) MTJ proximal to the injury site; and 3) MTJ distal to the injury site. The joint, synovium, and bursa were also evaluated (see appendix 3). Of note, the following descriptions are for the injured right hind limbs, with no pathologies appreciated in the sham left hind limbs at all time-points (score of 0 for all parameters).

Overall, the most significant and consistent qualitative finding was a pathologic fibrotic adhesive lesion at the muscle and tendon interface along the anterior/cranial aspect of the patella (figure 4) at the MTJ distal to the injury site. This lesion typically measured 25-400 um and was composed of reactive fibroblasts and disorganized collagen fibers, variably prominent myxomatous ground substance stroma, and vascular profiles. When focusing on individual parameters that may contribute to these findings, lesion size ( $\mathrm{p}=$ $0.34)$, vascularity $(\mathrm{p}=0.33)$, and fibrosis $(\mathrm{p}=0.06)$ at the MTJ distal to the injury site were not significantly different between time-points. However, the ground substance ( $\mathrm{p}$ $=0.02$ ) (see appendix 4) score was significant at the MTJ distal to the injury site, suggesting that this parameter may have the greatest contribution to the injury response and the overall pathology score.

In spite of the finding that the MTJ distal to the injury site progressively worsened over time, no significant differences were appreciated for the total injury score (figure $5 \mathrm{~F}, \mathrm{p}=$ 0.18 ), the MTJ proximal to the injury site (figure $5 \mathrm{~A}, \mathrm{p}=$ 0.10 ), MTJ injury site (figure $\mathbf{5} \mathbf{B}, \mathrm{p}=0.54$ ), or MTJ distal to the injury site (figure $5 \mathrm{C}, \mathrm{p}=0.11$ ). When considering the individual components which made up these scores, 


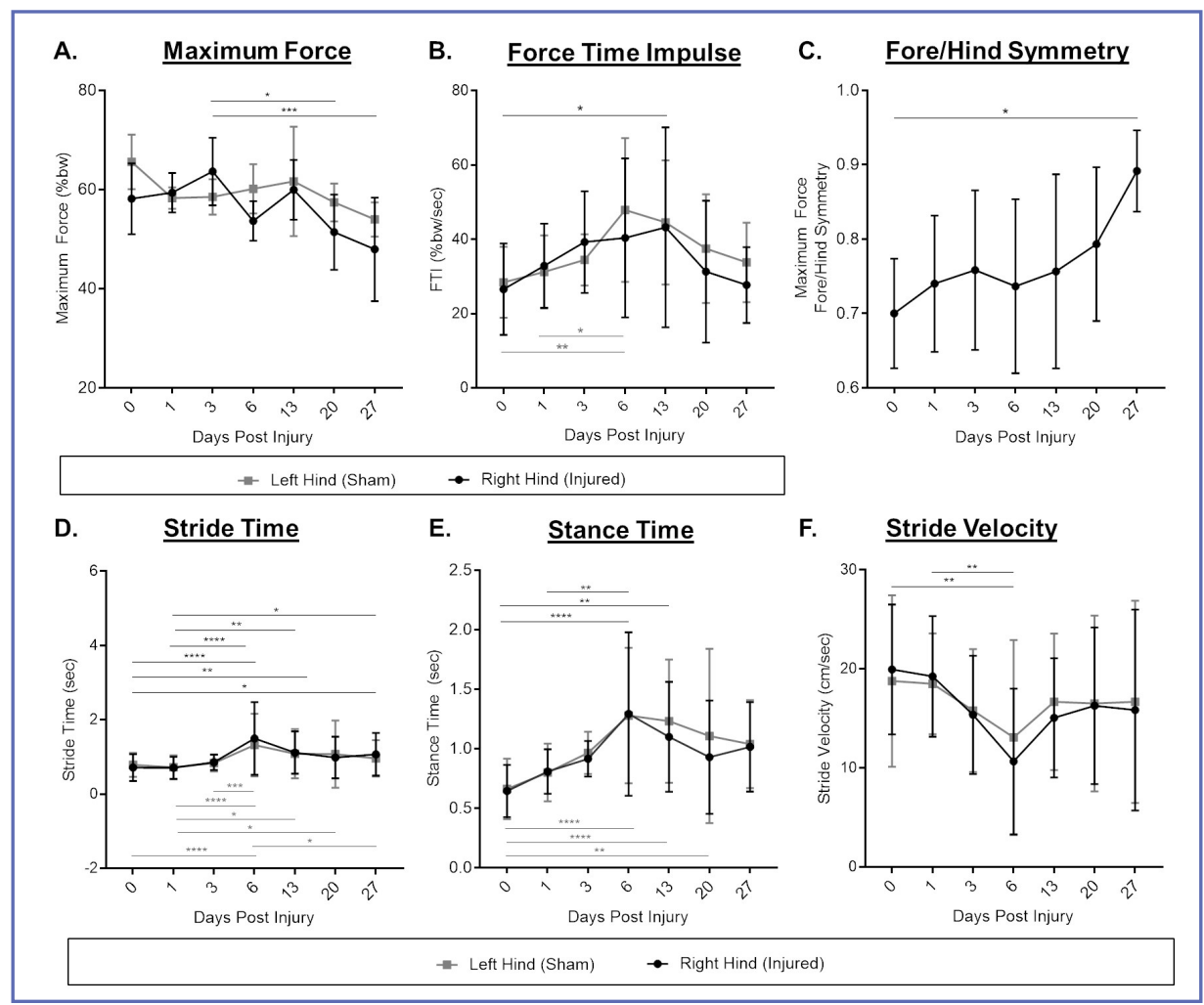

Figure 3. Voluntary weight bearing and gait parameters (Tekscan Rodent Walkway System) following quadriceps MTJ injury in the right hind limbs of rats. Significant parameters over time include (A) maximum force (\% body weight (bw)), (B) force time impulse (FTI; \% bw/sec), (C) maximum force fore/hind symmetry, (D) stride time (sec), (E) stance time (sec), and (F) stride velocity. Significance differences over time marked $\left({ }^{*} p<0.05,{ }^{* \star} p<0.01,{ }^{\star \star *} p<0.001,{ }^{* \star *} p<0.0001\right)$ and no statistically relevant between limb differences were noted. Values are represented as Mean +/- Standard Deviation with right hind (injured) differences in black at the top and left hind (sham) differences in grey at the bottom.

no significant differences in lesion size, fibrosis, ground substance, and vascularity were appreciated at the MTJ injury site. For the MTJ proximal to injury site, a significant decrease in fibrosis ( $\mathrm{p}=0.02$; between 1 day post-injury and both 7 and 28 days post-injury) was seen, with no significant changes in lesion size $(p=0.15)$, vascularity $(p=0.20)$, and ground substance. It was observed that the changes at the MTJ injury site and MTJ proximal to the needle site were subtle and the biological relevance of these findings remains undetermined.

With respect to secondary pathology changes, no significant differences between time-points were appreciated for the synovium ( $\mathrm{p}=0.84)$. However, significant differences were appreciated for joint space effusion (figure $4 \mathrm{D}$ and see appendix 5) and bursa (figure $4 \mathrm{E}$ and see appendix 6), although the pathology at both these sites seemed to decrease over time.

\section{DISCUSSION}

This animal model utilized needle tension to produce strain along the tendon-MTJ-muscle interface, a common site of impairment clinically $(2,3)$. Importantly, this work has resulted in a chronic model (up to 28 days post-injury) that histologically recapitulates muscle lesions adjacent to the MTJ in contrast to published models which focus on acute muscle injury (24-26). Therefore, this translational model of MTJ injury can be used for the study of aberrant or delayed healing and chronic scarring that may lead to high re-injury rates. Importantly, this strain-induced model also results in mobility changes associated with injured limb disuse, which can be used to study how therapeutics and rehabilitation strategies not only effect injury pathology but also mitigate decreased activity related to pain in clinical populations.

The major symptom and chief complaint of patients with muscle injuries is altered and/or painful mobility, yet there are few pre-clinical models which study functional alter- 


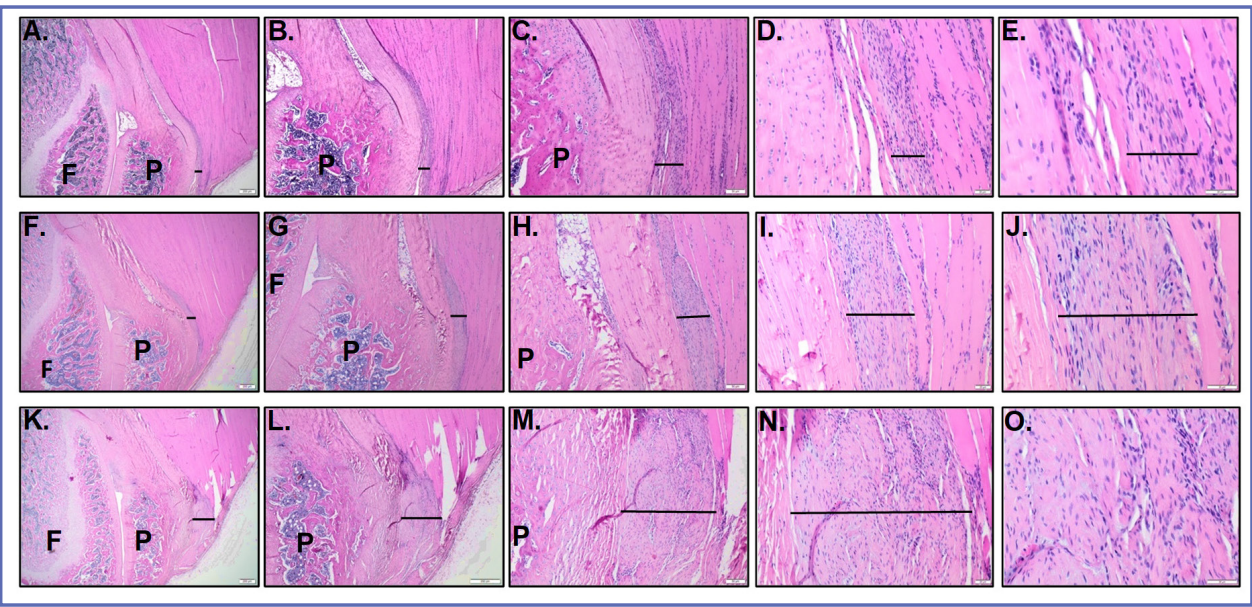

Figure 4. Representative photomicrographs of injury-associated pathology at the MTJ distal to the injury site. (A-E) Representative photomicrographs of an MTJ distal pathology score of 4. (A) Distal to the injury site along the anterior surface of the patella the MTJ and associated fascial planes are expanded by a linear proliferation of fibrous connective tissue, H\&E, 2X. (B) Higher magnification, H\&E, 4X. (C) The fibrous connective tissue proliferation measures $75 \mathrm{um}, \mathrm{H} \& \mathrm{E}, 10 \mathrm{X}$. (D) Higher magnification, H\&E, 20X. (E) Higher magnification, H\&E, 40X. (F-J) Representative photomicrographs of a MTJ distal pathology score of 7. (F) The MTJ distal to the injury site is expanded by a large adhesion composed of fibrous connective tissue, H\&E, 2X. (G) Higher magnification, H\&E, 4X. (H) The fibrous adhesion measures 100um, H\&E, 10X. (I) Higher magnification, H\&E, 20X. (J) Higher magnification, H\&E, 40X. (K-O) Representative photomicrographs of an MTJ distal pathology score of 9. (K) Distal to the injury site the MTJ is markedly expanded and effaced by haphazardly arranged fibrous connective tissue, H\&E, 2X. (L) Higher magnification, H\&E, 4X. (M) Fibrous connective tissue is accompanied by a myxomatous stroma which disrupt collagen fibers and support few vascular profiles, H\&E, 10X. (N) Higher magnification, H\&E, 20X. (O) Higher magnification, H\&E, 40X. F = femur; P = patella.

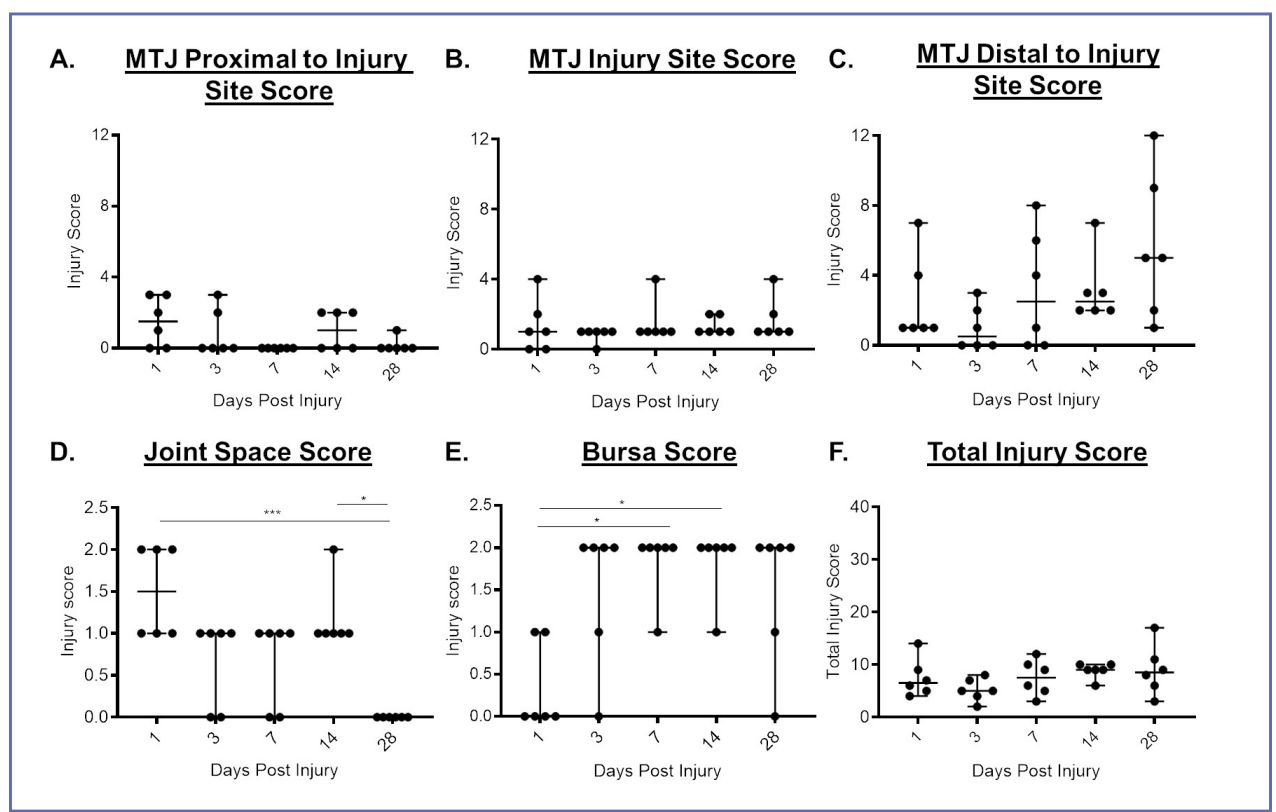

Figure 5. Individual MTJ Site Scores and Total MTJ Injury Score for the injured right hind limbs at 1, 3, 7, 14, and 28 days post-surgery. Injury scores for (A) MTJ proximal to the injury site, (B) MTJ at the injury site, (C) MTJ distal to injury site, as well as secondary changes within the (D) joint space and (E) bursa. (F) Total MTJ injury score reflects the sum of all pathology scores, with the maximal/maximum range of the scoring scheme provided on the $y$ axis. Values are represented as Median with Range and individual animals marked. Significant differences over time marked $\left({ }^{\star} p<0.05,{ }^{* \star} p<0.01,{ }^{* \star *} p<0.001,{ }^{\star * \star *} p<0.0001\right)$. 
ations in gait and/or weight bearing. Many studies focus on changes to muscle force production $(22,24-26)$; however, a direct association to limb alterations has not been established. Indeed, previous studies have shown that locomotive changes following injury precede muscle force changes (20), suggesting that re-establishment of functional mobility may initiate muscle activation and subsequent force production. Therefore, mobility measures may more accurately demonstrate early symptom modifications post-injury. Mobility in pre-clinical models can be studied using spatial (position-based), temporal (time-based), or kinetic (force-based) methods. To the authors' knowledge, this is the first study quantitatively analyzing all forms of functional mobility alterations using both voluntary and compulsory methods (temporal, spatial, and weight bearing) in an animal model of MTJ injury. In this study, the longitudinal tracking of individual animals throughout the experimental time-course using multiple methods has provided a comprehensive understanding of clinical manifestations of rectus femoris-specific MTJ injury.

With respect to temporal and spatial movement parameters, there were decreases in temporal parameters with voluntary movement (decreased velocity and increased stance time and stride time which peaks at 6 days post-injury) in the current model. Indeed, non-invasive kinetic methods to evaluate weight bearing distribution have been shown to sensitively predict limb dysfunction post-injury (34). With respect to weight bearing, subjective gait scoring following muscle contusion injury demonstrated decrease limb loading in a mouse model (21). As a less severe model was utilized in the current study, quantitative dynamic weight bearing was undertaken, and similar results were obtained. Rats placed less weight on their hind limbs over time (decreased maximum force and increased force time impulse) and shifted their weight to their forelimbs (increased maximum force for fore/ hind limb symmetry). Clinically, rectus femoris injury of the quadriceps muscle group typically occurs during the swing phase due to eccentric contractions of the muscle that occur during that portion of the gait cycle (4). Following rectus femoris MTJ injury in this study, animals were avoiding limb rotation (swing phase), which was associated with decreased animal movement. Therefore, it is likely that animals post-injury are attempting to avoid this swing phase to minimize rectus femoris muscle contraction. Of note, the functional mobility changes seen in the injured hind limb are also occurring in the contralateral hind limb that received a sham surgery.

Interestingly, no changes in temporal parameters were seen post-injury with compulsory treadmill-based gait analysis. Given the set treadmill speed and the fact that only data where the animal maintained speed with the treadmill were utilized for analysis, it can be difficult to appreciate natural gait differences. However, alterations in spatial parameters were documented with this obligatory method. Specifically, rats exhibited increased paw area and altered spatiotemporal parameters including increased Max dA/dt (impaired braking capacity). Collectively, our results suggest that following strain-induced MTJ injury, rats are favoring the stance phase to minimize limb rotation and movement during the swing phase. These results are corroborated by previous reports. Changes in ankle angle and calcaneus height have been shown in contusion models of anterior tibialis muscle injury (20), suggesting impaired joint rotation/movement. Additionally, voluntary walking impairment has previously been shown (19) in a rat model of traumatic muscle injury. In this study, treadmill-based walking trials were performed in triplicate to assess reproducibility in our clinical assessments. This necessitated 10 minutes of animal activity per timepoint, which was divided into short segments of non-strenuous compulsory movement followed by resting periods. We do not anticipate this level of movement modulated tissue remodeling in our model as more intense long term (> 60 minutes/day for 3-16 weeks) running protocols are typically utilized to induce muscle and tendon injury in rodents $(18,35,36)$. Along this vein, more intense running protocols may be employed in future investigations to assess if chronic MTJ scarring, a unique characteristic of this model, may lead to high re-injury rates.

To determine a relationship between the observed mobility alterations and histoanatomic manifestations of injury, we created a semi-quantitative grading scheme to characterize the inflammatory and structural response to MTJ injury throughout the experimental time-course. Our approach was to evaluate not only the MTJ but also nearby structures to gain a comprehensive understanding of how the entire region is responding to needle injury and to identify key contributors to injury progression. The grading scheme included evaluation of the needle puncture site, the region proximal and distal to the needle site, as well as nearby fat pads, bursa, synovial membranes, and knee joint spaces. Currently there is a lack of histopathologic data describing the acute and chronic manifestations of MTJ injuries in humans. Clinicians tend to rely on magnetic resonance imaging for injury evaluation and studies have demonstrated the presence of hemorrhage, edema, fatty infiltration, and muscle atrophy directly adjacent to the MTJ (6). Similarly, we have documented acute mixed inflammation and edema acutely after injury. In the later stages of healing, we see more chronic changes including: fibrosis, vascularization, and secondary changes to nearby structures including the joint space, bursae, and synovium.

The most striking and consistent qualitative histologic finding was a fairly large (50-300 um) adhesive lesion along the MTJ distal to the needle injury site 28 days post injury, 
which resulted in a significantly increased ground substance score at this time-point. It is anticipated that the development of this lesion was associated with an aberrant healing response resulting in early chronic injury progression (2). In regards to normal tissue healing, an initial injury (independent of severity) is followed by: (i) an inflammatory cell reaction and formation of a hematoma; (ii) phagocytosis of the damaged tissue (iii) repair and/or regeneration of myofibers; and lastly (iv) reorganization and remodeling with the aim of full functional recovery (2). Tissue regeneration at the MTJ can be particularly difficult due to the complex organization and anchoring between muscle myofibers and tendon collagen fibers (1). Indeed, in the current model, an excessive and disorganized fibroblastic response with diminished remodeling was seen as evidenced by the generation of a fibrotic scar 28 days post injury, which mimics the repair phase following muscle injury (2).

When identifying associations between histology and gait pattern outcome measures, it is noteworthy that the bursa score peaks at 7 days post-injury, which corresponds to decreases in voluntary movement at 6 days post-injury. Therefore, this may be a critical timeframe to target the local microenvironment towards a reparative $v s$ delayed/dysregulated healing process. Cellular and tissue level variations at this time-point may identify pathways that could be manipulated for organized healing. Future work is needed to investigate the functional role of these structures and the influence they may have on resident progenitor cells such as skeletal muscle satellite cells, fibroadipogenic precursors, interstitial tenocytes, fibroblasts, nerves, endothelial cells and inflammatory cells. As many of these cells types were present in both injured and sham limbs natively and at different time points post-injury their specific role in driving the histoanatomic manifestations of injury progression requires further study.

Rats were chosen due to their large size (relative to mice), inherent exercise ability for mobility outcomes, and the fact that the $\mathrm{Crl}: \mathrm{CD}(\mathrm{SD})$ rats are an outbred strain. Weight and skeletal measurements taken during the experimental time-course also demonstrate that rats were still growing, with skeletal maturity obtained at 16 weeks of age. Therefore, the results presented here are particularly relevant for adolescents and young adults (13-22 years of age) who

\section{REFERENCES}

1. Charvet B, Ruggiero F, Le Guellec D. The development of the myotendinous junction. A review. Muscles Ligaments Tendons J 2012;2(2):53-63.

2. Jarvinen TA, Jarvinen TL, Kaariainen M, Kalimo H, Jarvinen M. Muscle injuries: biology and treatment. Am J Sports Med 2005;33(5):745-64. experience MTJ associated sports-injuries. Notably, multiple prospective studies have identified that age is not a risk factor for quadriceps MTJ specifically, unlike in other MTJ etiologies $(14,37,38)$. Future studies will determine whether age plays a significant role in quadriceps MTJ injury and healing with this model.

Caveats of this study include the use of the contralateral limb as a control for both mobility and histopathological assessments. While this was undertaken due to clinical applicability, no significant changes between the injured right hind and contralateral sham limb were appreciated. Therefore, further study on the compensatory effects in quadruped animals is warranted to fully characterize this model. Additionally, all outcomes demonstrate sustained changes out to 28 days post-injury. Future directions include characterizing the injury at later (56 and 120 day) time-points to evaluate histologic changes (we suspect there will be adhesion contracture and remodeling) and mobility alterations later on throughout the repair process. Based on our findings out to 28 days post-injury, we anticipate that, while mobility measurements may normalize at 56 and 120 day timepoints, pathologic and scored features may be more distinct during these later stages of chronic tissue remodeling. The authors acknowledge that the exact strain which was induced during injury was not directly measured. However, the use of an $18 \mathrm{G}$ needle provided consistency among animals and dictated the degree of tissue plane separation as no excessive force was utilized.

The results from this study demonstrate the development of a chronic model of rectus femoris MTJ injury, altered mobility, and strain-induced fibrotic scarring along the anterior patella. Notably, these pathologic and functional changes recapitulate the course of injury progression similar to what is described in humans $(4,27,28)$. Collectively, this work provides a unique pre-clinical model to study quadriceps MTJ injury mechanisms for the identification of enhanced treatment options for patients who suffer from such activity-related muscle conditions.

\section{CONFLICT OF INTERESTS}

The authors declare that they have no conflict of interests.
3. Garrett WE, Jr. Muscle strain injuries. Am J Sports Med 1996;24(6 Suppl):S2-8.

4. Mendiguchia J, Alentorn-Geli E, Idoate F, Myer GD. Rectus femoris muscle injuries in football: a clinically relevant review of mechanisms of injury, risk factors and preventive strategies. Br J Sports Med 2013;47(6):359-66. 
5. Proske U, Allen TJ. Damage to skeletal muscle from eccentric exercise. Exerc Sport Sci Rev 2005;33(2):98-104.

6. Bencardino JT, Rosenberg ZS, Brown RR, Hassankhani A, Lustrin ES, Beltran J. Traumatic musculotendinous injuries of the knee: diagnosis with MR imaging. Radiographics 2000;20 Spec No:S103-20.

7. Jones BH, Cowan DN, Tomlinson JP, Robinson JR, Polly DW, Frykman PN. Epidemiology of injuries associated with physical training among young men in the army. Med Sci Sports Exerc 1993;25(2):197-203.

8. Davidson PL, Chalmers DJ, Wilson BD, McBride D. Lower limb injuries in New Zealand Defence Force personnel: descriptive epidemiology. Aust N Z J Public Health 2008;32(2):167-73.

9. Almeida SA, Williams KM, Shaffer RA, Brodine SK. Epidemiological patterns of musculoskeletal injuries and physical training. Med Sci Sports Exerc 1999;31(8):1176-82.

10. Drakos MC, Domb B, Starkey C, Callahan L, Allen AA. Injury in the national basketball association: a 17-year overview. Sports Health 2010;2(4):284-90.

11. Shi J, Gardner S, Wheeler KK, et al. Characteristics of nonfatal occupational injuries among U.S. workers with and without disabilities. Am J Ind Med 2015;58(2):168-77.

12. Noonan TJ, Garrett WE, Jr. Muscle strain injury: diagnosis and treatment. J Am Acad Orthop Surg 1999;7(4):262-9.

13. Kaufman KR, Brodine S, Shaffer R. Military training-related injuries: surveillance, research, and prevention. Am J Prev Med 2000;18(3 Suppl):54-63.

14. Orchard JW. Intrinsic and extrinsic risk factors for muscle strains in Australian football. Am J Sports Med 2001;29(3):300-3.

15. Yan Z, Choi S, Liu X, et al. Highly coordinated gene regulation in mouse skeletal muscle regeneration. J Biologic Chem 2003;278(10):8826-36.

16. Mitchell CA, McGeachie JK, Grounds MD. Cellular differences in the regeneration of murine skeletal muscle: a quantitative histological study in SJL/J and BALB/c mice. Cell Tissue Res 1992;269(1):159-66.

17. Anderson SE, Han WM, Srinivasa V, et al. Determination of a Critical Size Threshold for Volumetric Muscle Loss in the Mouse Quadriceps. Tissue Eng Part C Methods 2019;25(2):59-70.

18. Thirupathi A, Freitas S, Sorato HR, et al. Modulatory effects of taurine on metabolic and oxidative stress parameters in a mice model of muscle overuse. Nutrition 2018:54:158-64.

19. Dos Santos LS, Saltorato JC, Monte MG, et al. PBMT and topical diclofenac as single and combined treatment on skeletal muscle injury in diabetic rats: effects on biochemical and functional aspects. Lasers Med Sci 2019;34(2):255-62.

20. Iwata A, Fuchioka S, Hiraoka K, Masuhara M, Kami K. Characteristics of locomotion, muscle strength, and muscle tissue in regenerating rat skeletal muscles. Muscle Nerve 2010;41(5):694-701.

21. Rahusen FT, Weinhold PS, Almekinders LC. Nonsteroidal anti-inflammatory drugs and acetaminophen in the treatment of an acute muscle injury. Am J Sports Med 2004;32(8):1856-9.

22. Pratt SJP, Lawlor MW, Shah SB, Lovering RM. An in vivo rodent model of contraction-induced injury in the quadriceps muscle. Injury 2012;43(6):788-93.
23. Liu M, Wang Z, Lee C, et al. Brown/Beige Fat Activation after Skeletal Muscle Ischemia-Reperfusion Injury. Muscles Ligaments Tendons Journal 2020;10(4):579-88.

24. Contreras-Munoz P, Fernandez-Martin A, Torrella R, et al. A New Surgical Model of Skeletal Muscle Injuries in Rats Reproduces Human Sports Lesions. Int J Sports Med 2016;37(3):183-90.

25. Best TM, McCabe RP, Corr D, Vanderby R, Jr. Evaluation of a new method to create a standardized muscle stretch injury. Med Sci Sports Exerc 1998;30(2):200-5.

26. Brickson SL, McCabe RP, Pala AW, Vanderby R, Jr. A model for creating a single stretch injury in murine biarticular muscle. BMC Sports Sci Med Rehabil 2014;6(1):14.

27. Speer KP, Lohnes J, Garrett WE, Jr. Radiographic imaging of muscle strain injury. Am J Sports Med 1993;21(1):89-95; discussion 6.

28. Hughes Ct, Hasselman CT, Best TM, Martinez S, Garrett WE, Jr. Incomplete, intrasubstance strain injuries of the rectus femoris muscle. Am J Sports Med 1995;23 (4):500-6.

29. Sonin AH, Fitzgerald SW, Bresler ME, Kirsch MD, Hoff FL, Friedman H. MR imaging appearance of the extensor mechanism of the knee: functional anatomy and injury patterns. Radiographics 1995;15(2):367-82.

30. Flores DV, Mejia Gomez C, Estrada-Castrillon M, Smitaman E, Pathria MN. MR Imaging of Muscle Trauma: Anatomy, Biomechanics, Pathophysiology, and Imaging Appearance. Radiographics 2018;38(1):124-48.

31. Costa AF, Di Primio GA, Schweitzer ME. Magnetic resonance imaging of muscle disease: a pattern-based approach. Muscle Nerve 2012;46(4):465-81.

32. Padulo J, Oliva F, Frizziero A, Maffulli N. Muscles, Ligaments and Tendons Journal - Basic principles and recommendations in clinical and field Science Research: 2018 update. Muscles Ligaments Tendons J 2018;8(3):305-7.

33. Faul F, Erdfelder E, Lang AG, Buchner A. G*Power 3: a flexible statistical power analysis program for the social, behavioral, and biomedical sciences. Behav Res Methods 2007;39(2):175-91.

34. Pardes AM, Freedman BR, Soslowsky LJ. Ground reaction forces are more sensitive gait measures than temporal parameters in rodents following rotator cuff injury. J Biomech 2016;49(3):376-81.

35. Rooney SI, Loro E, Sarver JJ, et al. Exercise protocol induces muscle, tendon, and bone adaptations in the rat shoulder. Muscles Ligaments Tendons J 2014;4(4):413-9.

36. Jafari L, Vachon P, Beaudry F, Langelier E. Histopathological, biomechanical, and behavioral pain findings of Achilles tendinopathy using an animal model of overuse injury. Physiol Rep 2015;3(1).

37. Ekstrand J, Hagglund M, Walden M. Epidemiology of muscle injuries in professional football (soccer). Am J Sports Med 2011;39(6):1226-32.

38. Bradley PS, Portas MD. The relationship between preseason range of motion and muscle strain injury in elite soccer players. J Strength Cond Res 2007;21(4):1155-9. 


\section{SUPPLEMENTS}

Appendix 1. Comparison of published rodent and rabbit animal models targeting muscle injury.

\begin{tabular}{|c|c|c|c|c|c|c|}
\hline \multirow[t]{2}{*}{ Reference } & \multirow[t]{2}{*}{ Species } & \multirow[t]{2}{*}{ Injury Induction } & \multicolumn{4}{|c|}{ Model Characteristics } \\
\hline & & & Muscle Group & $\begin{array}{l}\text { Targets MTJ } \\
\text { (Y/N) }\end{array}$ & $\begin{array}{l}\text { Muscle } \\
\text { Lesion (Y/N) }\end{array}$ & $\begin{array}{l}\text { Muscle Lesion Resolution } \\
\text { Time-Point (days) }\end{array}$ \\
\hline (15) & Mouse & Chemical & Tibialis Anterior & $\mathrm{N}$ & $\mathrm{Y}$ & 14 days \\
\hline (16) & Mouse & Traumatic & Tibialis Anterior & $\mathrm{N}$ & $\mathrm{Y}$ & 6-10 days \\
\hline (17) & Mouse & Traumatic & Quadriceps & $\mathrm{N}$ & $\mathrm{Y}$ & Not Determined, $>28$ days \\
\hline (18) & Mouse & $\begin{array}{l}\text { Acute High } \\
\text { Intensity Exercise }\end{array}$ & Quadriceps & $\mathrm{N}$ & $\mathrm{N}$ & $\mathrm{N} / \mathrm{A}$ \\
\hline (19) & Rat & Contusion & Tibialis Anterior & $\mathrm{N}$ & $\mathrm{N}$ & $\mathrm{N} / \mathrm{A}$ \\
\hline (20) & Rat & Contusion & Gastrocnemius & $\mathrm{N}$ & $\mathrm{Y}$ & 28 days \\
\hline (21) & Mouse & Contusion & Tibialis Anterior & $\mathrm{N}$ & $\mathrm{Y}$ & Not Determined, $>7$ days \\
\hline (22) & Mouse/Rat & Contraction & Quadriceps & $\mathrm{N}$ & $\mathrm{Y}$ & 8 days \\
\hline (23) & Mouse & $\begin{array}{l}\text { Ischemia- } \\
\text { Reperfusion }\end{array}$ & Gastrocnemius & $\mathrm{N}$ & $\mathrm{Y}$ & 28 days \\
\hline (24) & Rat & Traumatic & Gastrocnemius & $\mathrm{Y}$ & $\mathrm{Y}$ & 26-46 days \\
\hline$(25)$ & Rabbit & Mechanical & Tibialis Anterior & $\mathrm{Y}$ & $\mathrm{N}$ & N/A \\
\hline (26) & Mice & Tetanic & Gastrocnemius & $\mathrm{Y}$ & $\mathrm{N}$ & $\mathrm{N} / \mathrm{A}$ \\
\hline Current & Rat & Strain & Quadriceps & $\mathrm{Y}$ & $\mathrm{Y}$ & Not Determined, $>28$ days \\
\hline
\end{tabular}

Grey shading denotes model characteristics that match the current model.

Appendix 2. Representative image of the generation of strain-induced MTJ injury in the quadriceps.

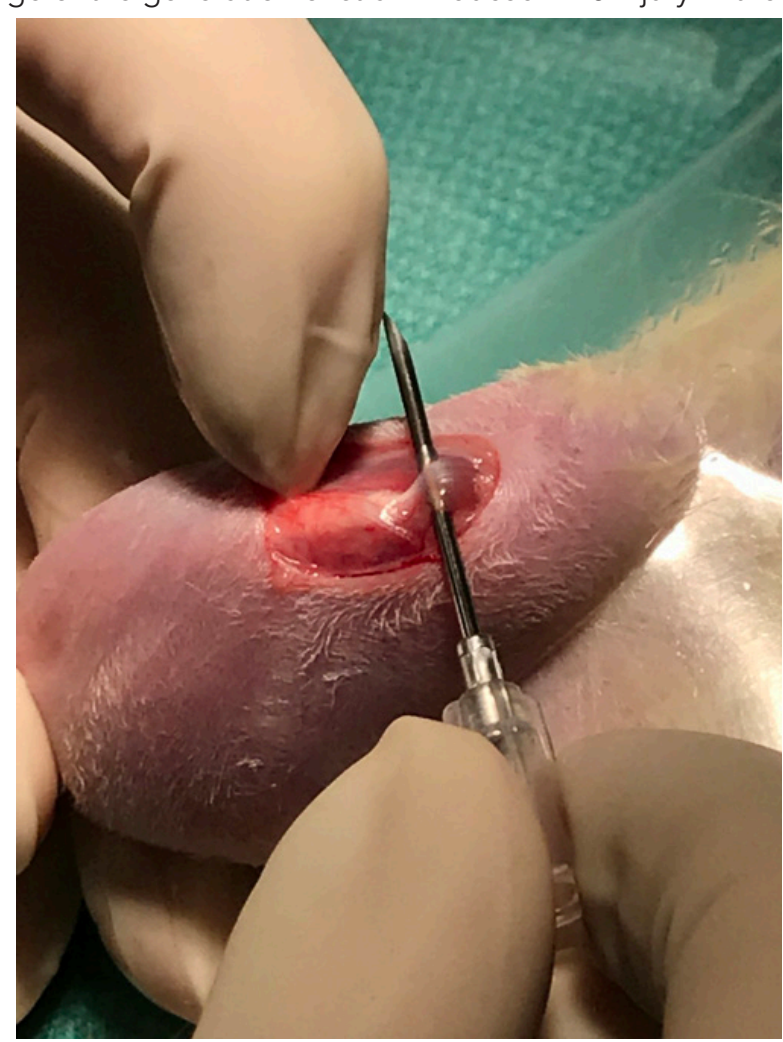


Appendix 3. Histopathologic grading scale for quadriceps MTJ injury pathology. The MTJ injury site, MTJ proximal to the injury site, and MTJ distal to the injury site were each graded independently on a scale of 0-3pts, with a maximum score of 12 possible for each site. The synovium, bursa, and joint were graded separately on a scale of 0-2pts each. Total MTJ injury score consisted of the sum of all sites and parameters with a maximum score of 41 possible.

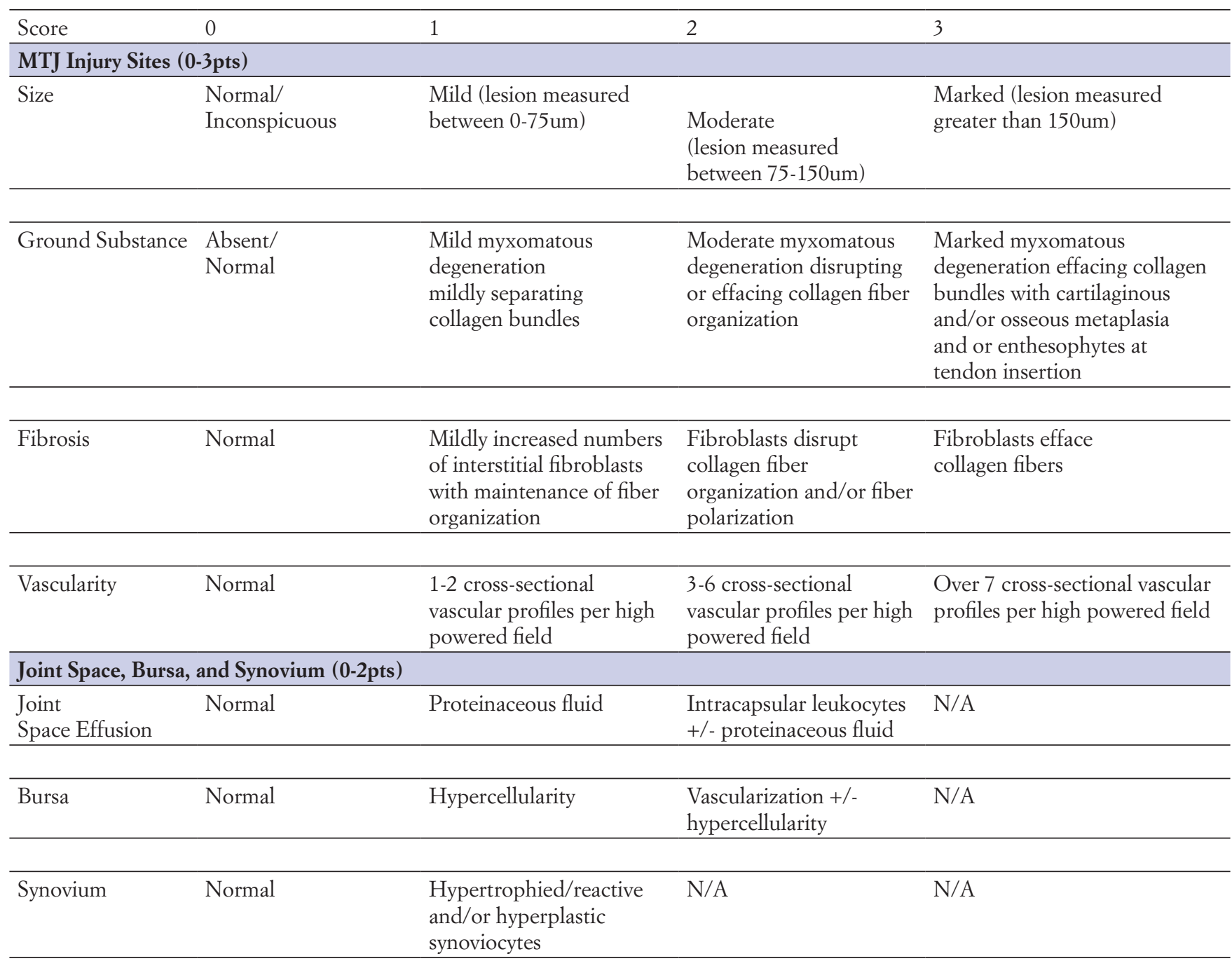




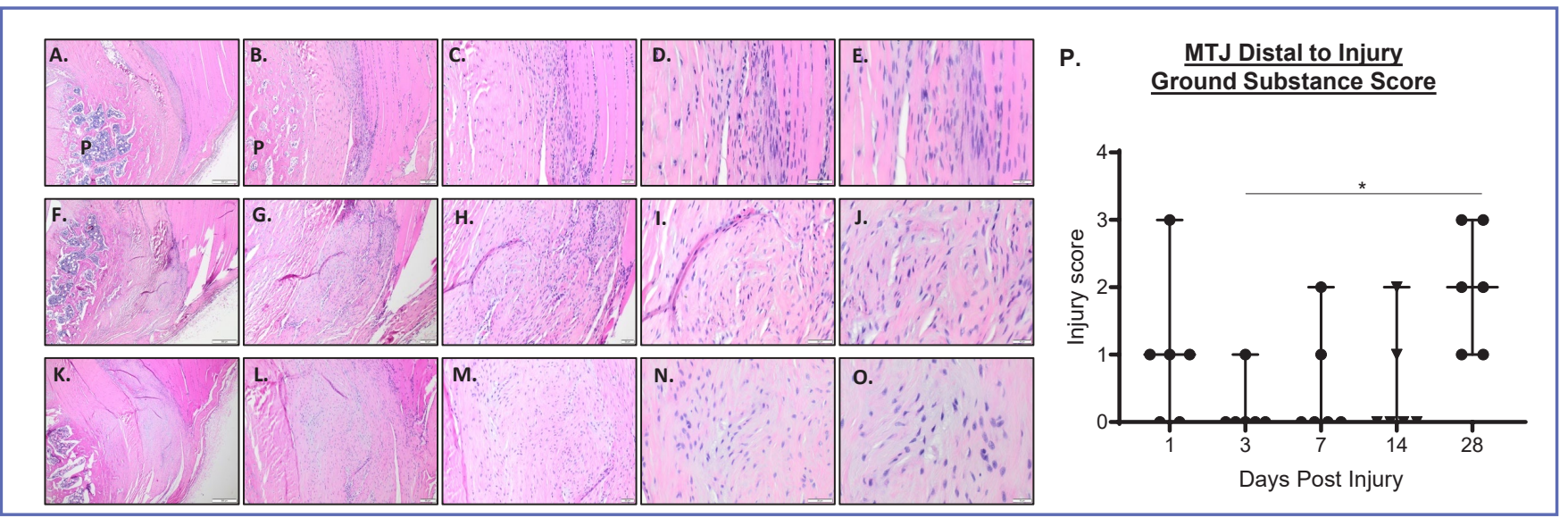

Appendix 4. Representative photomicrographs of MTJ ground substance pathology distal to the injury site. (A-E) Representative photomicrographs of MTJ ground substance score of 1. (A) Distal to the needle injury site along the anterior surface of the patella (marked with P) the MTJ is expanded by a linear proliferation of fibrous connective tissue which is accompanied by a mild basophilic myxomatous stroma, H\&E, 4X. (B) Higher magnification, H\&E, 10X. (C) Higher magnification, H\&E, 20X. (D) Higher magnification, H\&E, 40X. (E) Myxomatous degeneration mildly separates collagen fibers, H\&E, 60X. (F-J) Representative photomicrographs of MTJ ground substances score of 2. (F) The MTJ distal to the injury site is effaced by a bed of fibrous connective tissue, H\&E, 4X. (G) Higher magnification, H\&E, 10X. (H) Higher magnification, H\&E, 20X. (I) Higher magnification, H\&E, 40X. (J) Fibrous connective tissue is accompanied by a moderate amount of myxomatous ground substance, H\&E, 60X. (K-O) Representative photomicrographs of MTJ ground substance score of 3. (K) The MTJ distal to the injury site is markedly expanded and effaced by haphazardly arranged fibrous connective tissue, H\&E, 4X. (L) Higher magnification, H\&E, 10X. (M) Fibrous connective tissue is accompanied by a prominent basophilic myxomatous stroma, H\&E, 20X. (N) Higher magnification, H\&E, 40X. (O) Higher magnification, H\&E, 60X. (P) MTJ distal to the injury site ground substance scores for the injured right hind limbs at $1,3,7,14$, and 28 days post-surgery. Values are represented as Median with Range and individual animals marked. Significant differences over time marked $\left({ }^{\star} p<0.05,{ }^{* \star} p<0.01,{ }^{* \star *} p<0.001,{ }^{* \star * \star} p<0.0001\right)$.

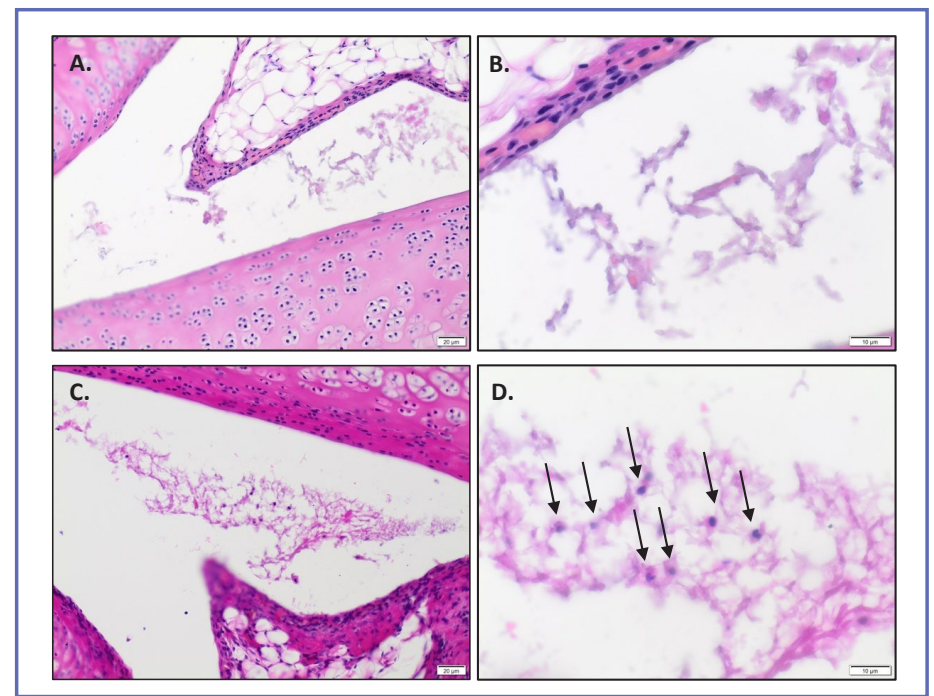

Appendix 5. Representative photomicrographs of joint effusion pathology. (A-B) Representative photomicrographs of joint effusion score of 1. (A) Within the joint space is a moderate amount of eosinophilic proteinaceous fluid, H\&E, 20X. (B) Higher magnification, H\&E, 60X. (C-D) Representative photomicrographs of joint effusion score of 2. (C) Within the joint space is a moderate amount of eosinophilic proteinaceous fluid which supports few variably degenerate leukocytes (arrows), H\&E, 20X. (D) Higher magnification, H\&E, 60X. 

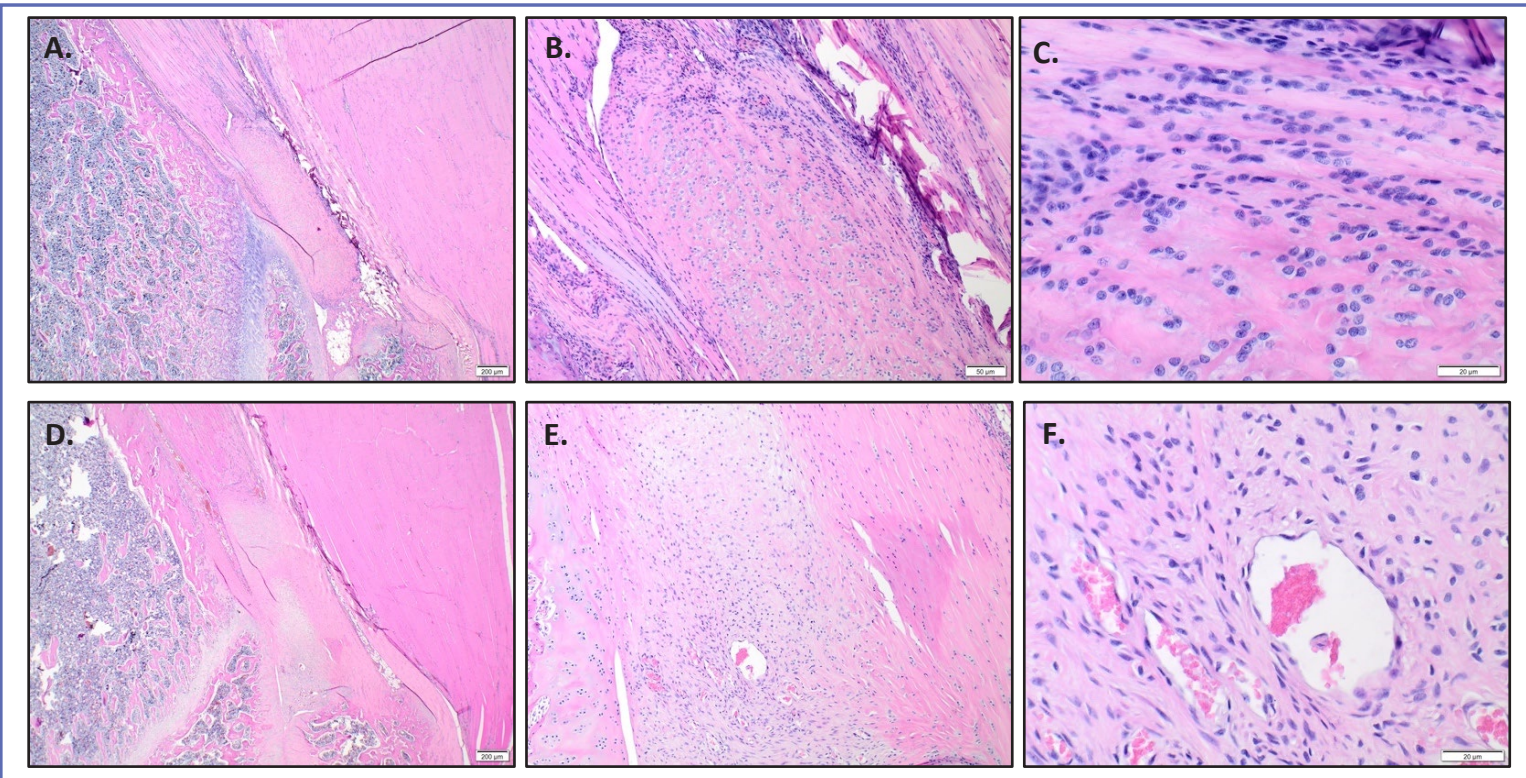

Appendix 6. Representative photomicrographs of bursal pathology. (A-C) Representative photomicrographs of bursal pathology score of 1. A. The proximal, anterior, and distal aspect of the bursa is hypercellular, H\&E, 2X. (B) Higher magnification, H\&E, 10X. (C) Higher magnification, H\&E, 40X. (D-F) Representative photomicrographs of bursal pathology score of 2. (D) The distal and proximal aspect of the bursal contains increased numbers of reactive spindle cells accompanied by moderate numbers of vascular profiles, H\&E, 2X. (E) Higher magnification, H\&E, 10X. (F) Higher magnification, H\&E, 40X. 